Marquette University

e-Publications@Marquette

Biomedical Engineering Faculty Research and Publications

Biomedical Engineering, Department of

$2-2021$

\title{
Somatosensory Deafferentation Reveals Lateralized Roles of Proprioception in Feedback and Adaptive Feedforward Control of Movement and Posture
}

\author{
S.A.L. Jayasinghe \\ Pennsylvania State University College of Medicine \\ F. R. Sarlegna \\ Aix Marseille Université \\ Robert A. Scheidt \\ Marquette University, robert.scheidt@marquette.edu \\ R. L. Sainburg \\ Pennsylvania State University College of Medicine
}

Follow this and additional works at: https://epublications.marquette.edu/bioengin_fac

Part of the Biomedical Engineering and Bioengineering Commons

\section{Recommended Citation}

Jayasinghe, S.A.L.; Sarlegna, F. R.; Scheidt, Robert A.; and Sainburg, R. L., "Somatosensory Deafferentation Reveals Lateralized Roles of Proprioception in Feedback and Adaptive Feedforward Control of Movement and Posture" (2021). Biomedical Engineering Faculty Research and Publications. 630.

https://epublications.marquette.edu/bioengin_fac/630 
Marquette University

e-Publications@Marquette

\section{Biomedical Engineering Faculty Research and Publications/College of} Engineering

This paper is NOT THE PUBLISHED VERSION.

Access the published version via the link in the citation below.

Current Opinion in Physiology, Vol. 19 (February 2021): 141-147. DOI. This article is (C) Elsevier and permission has been granted for this version to appear in e-Publications@Marquette. Elsevier does not grant permission for this article to be further copied/distributed or hosted elsewhere without the express permission from Elsevier.

\section{Somatosensory Deafferentation Reveals Lateralized Roles of Proprioception in Feedback and Adaptive Feedforward Control of Movement and Posture}

Shanie A.L. Jayasinghe

Department of Neurology, Pennsylvania State University College of Medicine, Hershey, PA Fabrice R. Sarlegna Aix Marseille Univ, CNRS, ISM, Marseille, France

Robert A. Scheidt

Department of Biomedical Engineering, Marquette University and the Medical College of Wisconsin, Milwaukee, WI

Department of Physical Medicine and Rehabilitation, Northwestern University Feinberg School of Medicine, Chicago, IL

Robert L. Sainburg

Department of Neurology, Pennsylvania State University College of Medicine, Hershey, PA 
Proprioception provides crucial information necessary for determining limb position and movement, and plausibly also for updating internal models that might underlie the control of movement and posture. Seminal studies of upper-limb movements in individuals living with chronic, large fiber deafferentation have provided evidence for the role of proprioceptive information in the hypothetical formation and maintenance of internal models to produce accurate motor commands. Vision also contributes to sensorimotor functions but cannot fully compensate for proprioceptive deficits. More recent work has shown that posture and movement control processes are lateralized in the brain, and that proprioception plays a fundamental role in coordinating the contributions of these processes to the control of goal-directed actions. In fact, the behavior of each limb in a deafferented individual resembles the action of a controller in isolation. Proprioception, thus, provides state estimates necessary for the nervous system to efficiently coordinate multiple motor control processes.

\section{Introduction}

Proprioception, a term coined by Sir Charles Sherrington in 1906 [1], refers to information about position and movement derived from muscular, tendon and articular sensors. Later research emphasized the specific role of muscle spindles and Golgi tendon organs by demonstrating that joint position sense remains largely intact after complete joint replacement surgeries [2], a finding consistent with experimental demonstrations that joint receptors do not provide information in the midrange of the joint [3]. Rare cases of large fiber sensory neuropathy (LFSN) have shown that a massive, yet specific loss of the large afferent fibers that innervate Golgi tendon organs (Ib) and muscle spindles (la and II) result in a profound loss of position and movement sensation, regardless of intact small fibers that innervate joint articular tissues and skin. Since most energetically-costly sensory neurons (with large fibers) in the mammalian system innervate these sensors, and muscle spindles are found in all skeletal muscles in the primate system, this suggests an important role of proprioception in adaptive behavior.

Seminal research on these sensors, their innervation and central projections has elucidated the important roles of spinal circuits, including both homogenous and heterogenous reflexes, in modulating mechanical behavior of the muscles in response to perturbations [4,5]. Research in human deafferentation has introduced the important role of proprioception in feedforward mechanisms which are largely mediated by supraspinal centers, including the cerebellum [6] and regions of the cerebral cortex $\left[7^{\bullet}, 8,9\right]$. In this review, we elucidate the importance of proprioception in feedforward mechanisms that specify upper limb reaching trajectories and final limb positions, drawing largely on evidence from experimental studies in deafferented individuals with LFSN.

\section{The role of proprioception in updating 'internal models' for the feedforward control of movement}

Sensory information is used for both feedforward and feedback motor control processes (Figure 1).

Feedforward processes refer to the modification of system output using anticipated results in order to modify descending commands before the onset of the impending movement. Feedback processes 
compare state estimates derived from sensory signals to predicted sensory states, using the difference as an error signal to generate corrections. When the feedback loop is rapid and gains are optimized, feedback can lead to stability of responses, such as the linearization of the rapid and incremental stretch response by stiffness regulation [4]. However, errors requiring large proportional gains without an appropriate derivative gain, and invoking loops of longer latency, can lead to destabilizing responses. Feedforward mechanisms provide the ability to anticipate system responses to future output and, thus, allow for stability of behavior when predictions are fairly accurate. It should be stressed that feedforward mechanisms can be used to augment feedback systems, allowing robustness in the face of inaccurate predictions due to environmental variations, prediction errors, and/or neural noise (see Refs. $[10,11,12,13]$ for recent articles on optimal feedback control and gain modulation).

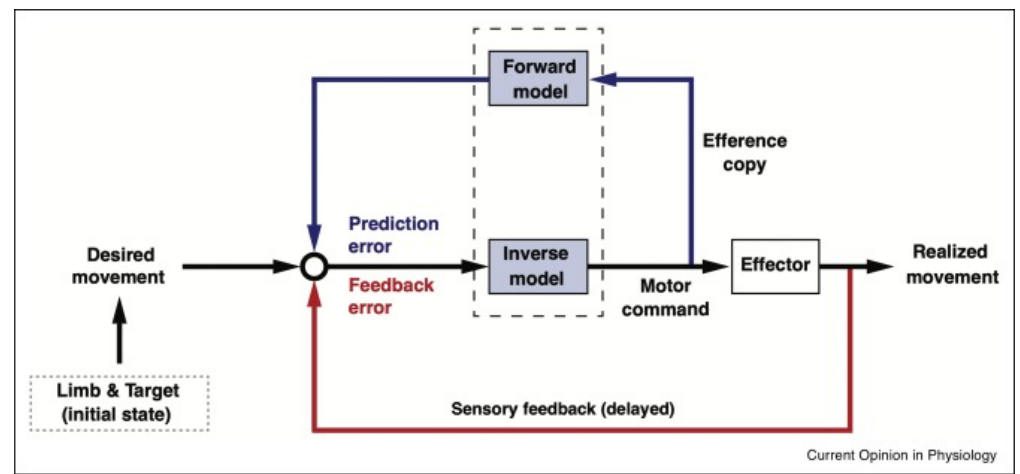

Figure 1. Internal models for feedforward and feedback motor control. An inverse model transforms differences between intended and actual limb configuration states into motor commands. These differences (errors) can be derived through pathways involving sensory feedback and pathways involving predictions of future states. Limb configuration information is derived from proprioception and/or vision. It can also be predicted using efference copy and the forward model.

The term 'internal model' has been widely studied through behavioral and neurophysiological research in the field of neuroscience $[14,15]$. Here we refer to an internal model as a neural process that allows prediction of motor actions and their consequences. The internal model is continuously updated by sensory information so that these predictions can be both accurate and useful. Predicted movement features vary depending on task goals and costs $[16,17]$. For instance, when kinematic and kinetic variables, such as smoothness and work, are critical costs for a task, the internal model makes predictions to optimize each variable $[18,19]$. While researchers can model this process through forward and inverse dynamic equations [20], we expect that the biological system predicts the effects of impending dynamic events in ways that do not require the specific mathematical formalisms implied by equations of motion. Thus, we emphasize that an internal model provides us with a computational foundation from which we can theorize about mechanisms of motor control. Cognitive models of the body, referred to as body schema, are also a type of internal model that appears to be dependent on proprioceptive information $[9,21,22]$. For example, Sacks has reported cases in which individuals who have lost proprioception can view their deafferented limbs as foreign and even pernicious [23].

Studies in deafferented individuals have demonstrated the critical roles played by proprioception and vision in the rapid online control of movement as well as in the development, maintenance and modification of internal models. There is experimental evidence that vision and proprioception may 
contribute differently to the control of movement direction and final positions [24, 25, 26, 27], but we do not currently know the extent to which each of these signals affects the action of theorized forward and inverse models of motor control. Vision can provide information about limb configuration, which may explain why deafferented individuals can adapt to a novel force field when provided with visual information about hand position, and update internal models used to predict the effects of limb dynamics using vision alone $[28,29,30]$.

While visual information also contributes to movement control, it does not suffice to maintain accuracy of the presumptive internal model that accounts for the effects of limb dynamics in specification of movement trajectories. Deafferented individuals make movements that are abnormal, even years after the proprioceptive loss [30,31,32${ }^{\circ}$, reflecting poor coordination of intersegmental dynamics [33,34]. A seminal study examining unconstrained multijoint movements in two deafferented individuals revealed their inability to accurately and sharply reverse movements of the arm when instructed to produce out-and-back goal-directed slicing motions of the hand [33]. The slicing motions required precise coordination of movement between shoulder and elbow joints, and were well achieved in neurologically intact controls; however, the deafferented individuals produced curved hand paths, rather than sharp movement reversals even when vision of the limb was provided during movement. This resulted from an inability to account for the intersegmental interaction torques produced at one joint by motion of the other joints.

A follow-up study [34] demonstrated that these reversal errors varied with the amplitude of such interaction torques. Participants made out-and-back movements along different directions and varying distances from the start position that required the same amount of elbow excursion but different amounts of shoulder excursion. The authors showed that deafferented individuals produced large reversal errors, which were unlike the sharp reversals made by control individuals (Figure 2a). A closer examination of limb dynamics during the reversal phase of the movement showed a statistically significant linear correlation between peak elbow acceleration and peak interaction torque in deafferented individuals, but not in control individuals (Figure 2b). This indicated that deafferented individuals were unable to maintain stable elbow motion because of direction-dependent variations in interaction torque. Therefore, hand path reversal errors in deafferented individuals resulted from discoordination of elbow and shoulder joint motion, in contrast to the tight interjoint coupling seen in control participants (Figure 2c). Because intersegmental interactions are transient and large in amplitude, interjoint coordination depends on feedforward mechanisms that shape motor output signals in accord with impending variations in movement direction and speed. This series of studies demonstrated the importance of proprioception in tuning the parameters used to predict and compensate for the effects of limb inertial dynamics on rapid arm movements. It is likely that feedforward and feedback processes share a model-based process that accounts for limb dynamics [35], and that proprioception provides the foundation necessary for coordinating posture and movement. 
(a)

Control: MFG

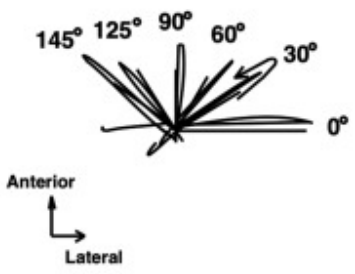

(b)

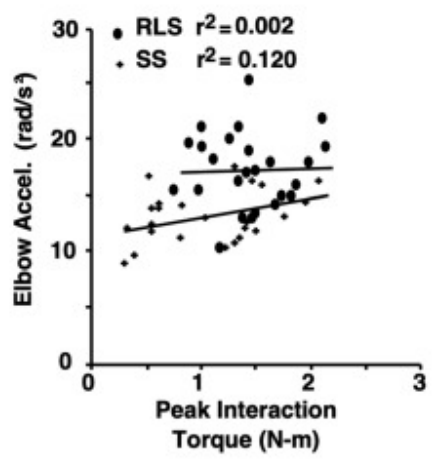

(c)

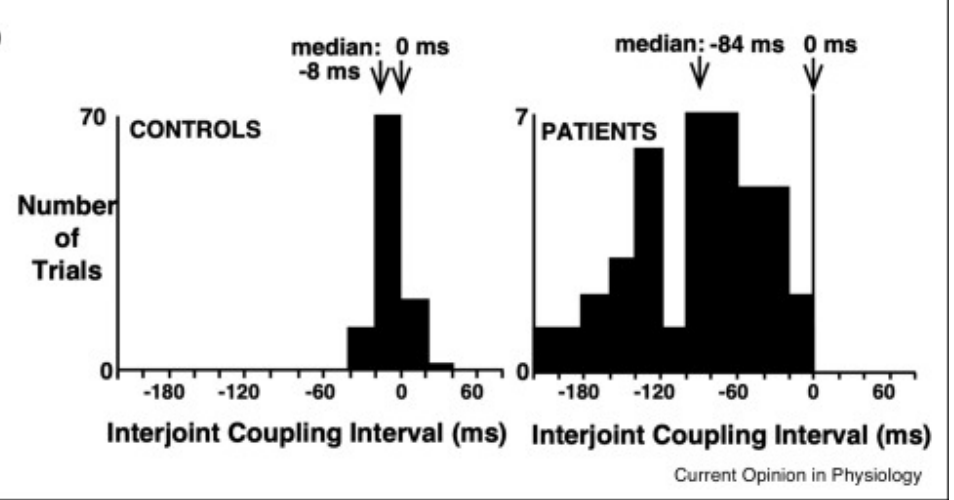

Patient: MA

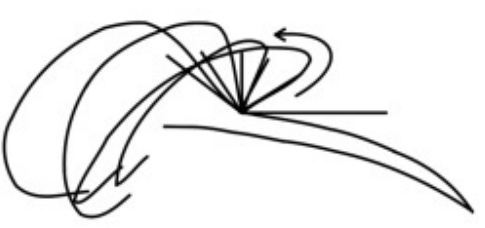

Patients

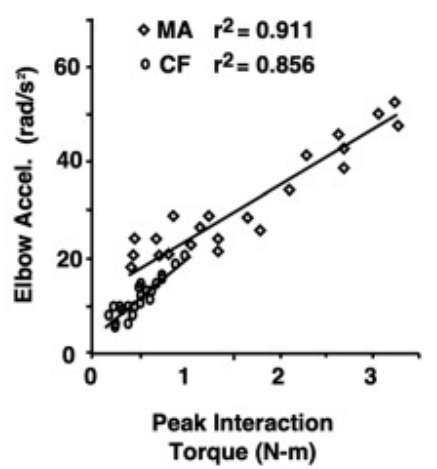

Current Opinion in Physiology

Figure 2. Loss of interjoint coordination in the absence of proprioception. (a) Representative hand paths from a control participant and a deafferented patient when completing an out-and-back tracing movement in 6 different directions. (b) Correlation between peak elbow joint acceleration and peak interaction torque during movement reversal for all 6 directions in two controls and two deafferented patients. (c) Histograms of the range of interjoint coupling intervals (time between elbow and shoulder reversals) for controls and deafferented patients. Adapted with permission from Ref. [34].

\section{Proprioception-mediated differential feedforward control of movement and}

\section{posture}

We now examine the crucial role of proprioception in the feedforward control of movement and posture. The coordination of the left and right arms in primates is most often asymmetric in ways suggesting that feedforward and/or feedback control are lateralized. The dynamic dominance hypothesis suggests a differentiation of the control of limb dynamics: in right-hand dominant 
individuals, the left hemisphere specializes in the feedforward control of trajectory dynamics and the right hemisphere specifies the feedforward and feedback control of limb impedance about final stable postures [36]. This hypothesis is in contrast with the prevailing view of handedness, the global dominance theory, which proposes that the dominant hand is superior in all aspects of performance [37]. However, our recent study on a deafferented individual (GL) revealed that proprioception does play a critical role in the lateralized control of both movement and posture mechanisms [38*0].

GL and a group of neurologically intact controls performed point-to-point reaching movements using each hand while the arm was supported on an air sled that eliminated the mechanical effects of gravity and friction. In the absence of vision of hand position, GL exhibited deficits in trajectory and final posture that differed between the left and right hand, and which differed markedly from movements of control participants (Figure 3a,b). GL's nondominant left hand produced large initial direction errors as well as oscillations at the target. Her dominant right hand produced less initial direction errors, but exhibited large drifts away from the target at the end of movement (Figure 3c,d). Computer simulations explained these findings as reflecting a temporal discoordination between separate and poorly tuned trajectory and impedance controllers, which signified abnormally low levels of damping and stiffness due to absent peripheral reflex activity. Previously, a serial hybrid model of movement explained differences in movement behavior between the left and right hands as resulting from differences in when control of the arm switches from predominantly trajectory control to predominantly impedance control [39].
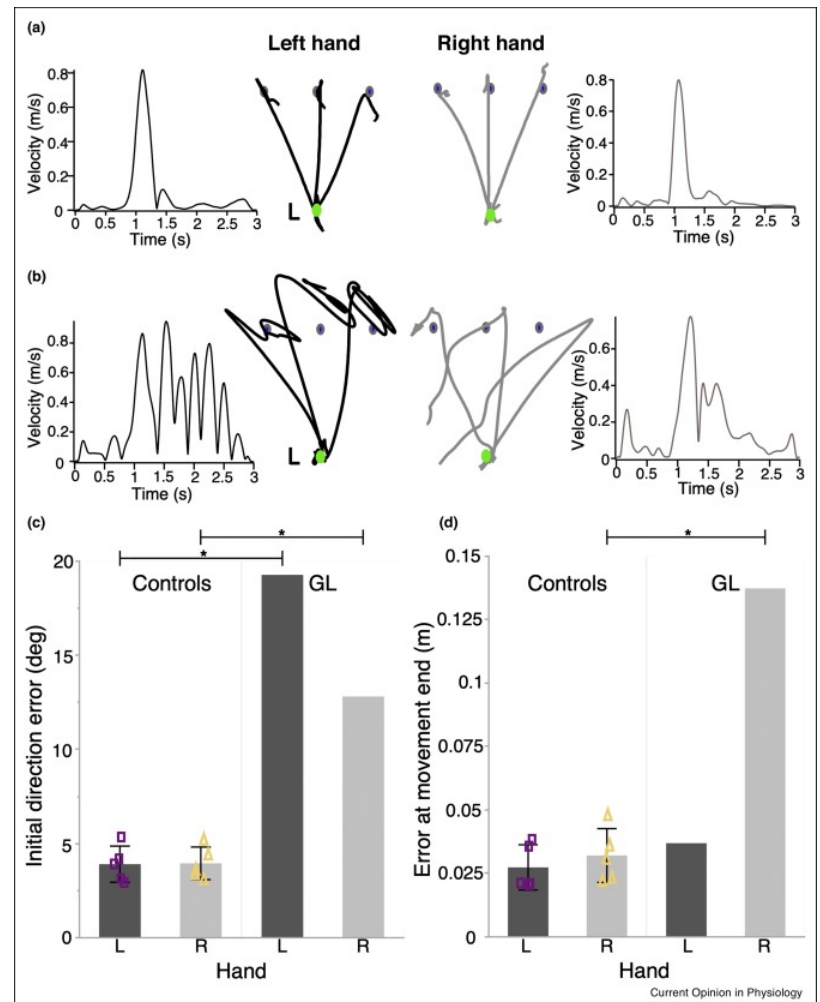

Figure 3. Deafferentation reveals differential deficits in the control of arm movement and posture in the two limbs. Participants performed reaching movements to a target (blue) placed in one of three directions from an initial start position (green). Vision of hand path was removed upon leaving the start position. Left and right hand paths and tangential velocity profiles are shown for (a) a representative control individual and (b) a deafferented individual. Scale bars next to the left hand trajectories represent $2 \mathrm{~cm}$ hand movement. (c) Mean 
initial direction error and (d) mean error at movement's end is displayed for each hand of 5 control participants and the deafferented individual. Error bars in control data represent 1 standard deviation from the mean. Mean values for each control participant are plotted as purple squares (left hand) or yellow triangles (right hand). $p<0.001\left(^{*}\right)$. Reprinted with permission from Ref. [38*0].

Experimental findings in GL and computer simulations are consistent with the idea that the switch time from trajectory to impedance control occurs earlier for the nondominant left hand than for the dominant right hand [38*0], thus suggesting an unequal contribution of each controller to each limb. Therefore, in the absence of online peripheral information, the limb that receives less direct input from a controller (i.e. the ipsilateral controller) would be especially impaired in the aspect(s) of performance for which the ipsilateral controller is specialized. The trajectories shown in Figure $3 \mathrm{~b}$ illustrate this point. Taken together, this line of research emphasizes the importance of proprioception in feedforward control processes used for coordination of movement trajectories and for stabilizing accurate final limb postures. This research also provides support for a complementary rather than global dominance view of handedness.

\section{Limitations and future directions}

The studies reviewed here mostly focus on reaching as a paradigm for studying human motor control in healthy and deafferented individuals. The deafferented patients have a specific, well-characterized proprioceptive loss; however, the experimental findings reflect the effects of both complete proprioceptive loss as well as the re-adaptation of motor skills to their chronic condition. While reaching is an important component of functional motor performance, many other actions are critical to adaptive behavior in humans, including but not limited to locomotor behaviors, object manipulation, catching and throwing behaviors, and coordination between whole body and limb movements for activities of daily living. The focus of the reviewed papers on reaching behaviors is an early step in understanding the role of proprioception in motor control and lateralization, which was driven by many decades of foundational research in motor control and biomechanics. Selecting a given paradigm is likely to influence the view of motor lateralization, which must be treated as a dynamic process where the contribution of each hemisphere to movement control is driven by the skill level and attentional focus of the performer [40], the type and complexity of the task, and the relative importance of the various goals and costs of the task [41]. The tasks employed to study the contributions of sensory information to motor control are likely to expand as our understanding of the basic principles of the sensory contributions to motor control advance. This should translate to innovations for restoring kinesthetic control (see Refs. $\left[42{ }^{*}, 43,44,45\right]$ ) in amputees and individuals with neurological disorders [46].

This short review of classical and recent work highlights the important role of proprioception in the feedforward control of trajectory and posture, and the integration of these processes to produce accurate movements.

\section{Conflict of interest statement}

Nothing declared. 


\section{References and recommended reading}

Papers of particular interest, published within the period of review, have been highlighted as:

- of special interest

$\bullet \bullet$ of outstanding interest

\section{Acknowledgements}

This work was supported by the National Institutes of Health [grant number R01HD059783 awarded to R.L.S.] and the National Center for Scientific Research-France (PICS grant awarded to F.R.S.).

\section{References}

1 C.S. Sherrington. Integrative Action of the Nervous System. The University Press (1906)

2 R.L. Barrack, H.B. Skinner, S.D. Cook, R. Haddad Jr. Effect of articular disease and total knee arthroplasty on knee joint-position sense. J Neurophysiol, 50 (1983), pp. 684-687

3 F.J. Clark, P. Burgess. Slowly adapting receptors in cat knee joint: can they signal joint angle? J Neurophysiol, 38 (1975), pp. 1448-1463

4 T. Nichols, J. Houk. Improvement in linearity and regulation of stiffness that results from actions of stretch reflex. J Neurophysiol, 39 (1976), pp. 119-142

5 C.M.J.I. Huyghues-Despointes, T.C. Cope, T.R. Nichols. Intrinsic properties and reflex compensation in reinnervated triceps surae muscles of the cat: effect of activation level. J Neurophysiol, 90 (2003), pp. 1537-1546

6 A.S. Therrien, A.J. Bastian. The cerebellum as a movement sensor. Neurosci Lett, 688 (2019), pp. 3740

7• E.H. de Haan, H.C. Dijkerman. Somatosensation in the brain: a theoretical re-evaluation and a new model. Trends Cogn Sci, 24 (2020), pp. 529-541. The authors present a review of somatosensory processing and a framework for a new model to describe the multiple interconnected neural pathways involved in various aspects of somatosensory function. They describe a 'cylinder block' consisting of the thalamus and the primary and secondary somatosensory areas that form the basic unit of somatosensory processing. Neuroimaging studies in humans reveal involvement of higher association areas that combine features of sensorimotor processes. The authors propose a distributed network of cortical and subcortical areas involved in somatosensation.

8 H. Ohashi, P.L. Gribble, D.J. Ostry. Somatosensory cortical excitability changes precede those in motor cortex during human motor learning. J Neurophysiol, 122 (2019), pp. 1397-1405

9 R.V. Bretas, M. Taoka, H. Suzuki, A. Iriki. Secondary somatosensory cortex of primates: beyond body maps, toward conscious self-in-the-world maps. Exp Brain Res, 238 (2020), pp. 259-272

10 S.H. Scott. Optimal feedback control and the neural basis of volitional motor control. Nat Rev Neurosci, 5 (2004), pp. 532-545

11 F. Crevecoeur, S.H. Scott, T. Cluff. Robust control in human reaching movements: a model-free strategy to compensate for unpredictable disturbances. J Neurosci, 39 (2019), pp. 8135-8148

12 J. Keyser, R.E. Ramakers, W.P. Medendorp, L.P. Selen. Task-dependent responses to muscle vibration during reaching. Eur J Neurosci, 49 (2019), pp. 1477-1490 
13 S. Franklin, D.M. Wolpert, D.W. Franklin. Rapid visuomotor feedback gains are tuned to the task dynamics. J Neurophysiol, 118 (2017), pp. 2711-2726

$14 \mathrm{M}$. Kawato. Internal models for motor control and trajectory planning. Curr Opin Neurobiol, 9 (1999), pp. 718-727

$15 \mathrm{~K}$. Vandevoorde, J.-J.O. de Xivry. Internal model recalibration does not deteriorate with age while motor adaptation does. Neurobiol Aging, 80 (2019), pp. 138-153

16 J.B. Heald, D.W. Franklin, D.M. Wolpert. Increasing muscle co-contraction speeds up internal model acquisition during dynamic motor learning. Sci Rep, 8 (2018), pp. 1-11

17 R. Shadmehr, M.A. Smith, J.W. Krakauer. Error correction, sensory prediction, and adaptation in motor control. Annu Rev Neurosci, 33 (2010), pp. 89-108

18 E. Burdet, R. Osu, D.W. Franklin, T.E. Milner, M. Kawato. The central nervous system stabilizes unstable dynamics by learning optimal impedance. Nature, 414 (2001), pp. 446-449

19 R. Shadmehr, F.A. Mussa-Ivaldi. Adaptive representation of dynamics during learning of a motor task. J Neurosci, 14 (1994), pp. 3208-3224

20 D.M. Wolpert, R.C. Miall, M. Kawato. Internal models in the cerebellum. Trends Cogn Sci, 2 (1998), pp. 338-347

$21 \mathrm{~J}$. Cole, J. Paillard. Living without touch and peripheral information about body position and movement: studies with deafferented subjects. The Body and the Self, The MIT Press (1995), pp. 245-266

22 M. Martel, L. Cardinali, G. Bertonati, C. Jouffrais, L. Finos, A. Farnè, A.C. Roy. Somatosensory-guided tool use modifies arm representation for action. Sci Rep, 9 (2019), pp. 1-14

23 O. Sacks. The Man Who Mistook His Wife for a Hat and Other Clinical Tales. Summit Books, New York (1985)

24 B.A. Shabbott, R.L. Sainburg. On-line corrections for visuomotor errors. Exp Brain Res, 195 (2009), pp. 59-72

25 R.A. Scheidt, C. Ghez. Separate adaptive mechanisms for controlling trajectory and final position in reaching. J Neurophysiol, 98 (2007), pp. 3600-3613

26 R.A. Scheidt, M.A. Conditt, E.L. Secco, F.A. Mussa-Ivaldi. Interaction of visual and proprioceptive feedback during adaptation of human reaching movements. J Neurophysiol, 93 (2005), pp. 3200-3213

27 C. Ghez, R. Scheidt, H. Heijink. Different learned coordinate frames for planning trajectories and final positions in reaching. J Neurophysiol, 98 (2007), pp. 3614-3626

28 F.R. Sarlegna, N. Malfait, L. Bringoux, C. Bourdin, J.-L. Vercher. Force-field adaptation without proprioception: can vision be used to model limb dynamics?. Neuropsychologia, 48 (2010), pp. 60-67

29 H.Z. Lefumat, R.C. Miall, J.D. Cole, L. Bringoux, C. Bourdin, J.-L. Vercher, F.R. Sarlegna. Generalization of force-field adaptation in proprioceptively-deafferented subjects. Neurosci Lett, 616 (2016), pp. 160-165

30 R.C. Miall, N.M. Kitchen, S.-H. Nam, H. Lefumat, A.G. Renault, K. Ørstavik, J.D. Cole, F.R. Sarlegna. Proprioceptive loss and the perception, control and learning of arm movements in humans: evidence from sensory neuronopathy. Exp Brain Res, 236 (2018), pp. 2137-2155 
31 C. Cuadra, A. Falaki, R. Sainburg, F.R. Sarlegna, M.L. Latash. Case studies in neuroscience: the central and somatosensory contributions to finger interdependence and coordination: lessons from a study of a "deafferented person". J Neurophysiol, 121 (2019), pp. 2083-2087

32 R.C. Miall, O. Rosenthal, K. Ørstavik, J.D. Cole, F.R. Sarlegna. Loss of haptic feedback impairs control of hand posture: a study in chronically deafferented individuals when grasping and lifting objects. Exp Brain Res, 237 (2019), pp. 2167-2184. The authors examined the functional consequences of haptic deficits by observing performance in three deafferented individuals on a reach-grasp-lift task that involved fine motor manipulation skills. This study showed that deafferented individuals tend to produce hand postures with reduced degrees of freedom in order to provide more stability to the grip. Hand posture variability is also high, especially when manipulating smaller objects.

33 R.L. Sainburg, H. Poizner, C. Ghez. Loss of proprioception produces deficits in interjoint coordination. J Neurophysiol, 70 (1993), pp. 2136-2147

34 R.L. Sainburg, M.F. Ghilardi, H. Poizner, C. Ghez. Control of limb dynamics in normal subjects and patients without proprioception. J Neurophysiol, 73 (1995), pp. 820-835

35 R.S. Maeda, T. Cluff, P.L. Gribble, J.A. Pruszynski. Feedforward and feedback control share an internal model of the arm's dynamics. J Neurosci, 38 (2018), pp. 10505-10514

36 R.L. Sainburg. Evidence for a dynamic-dominance hypothesis of handedness. Exp Brain Res, 142 (2002), pp. 241-258

$37 \mathrm{U}$. Ziemann, M. Hallett. Hemispheric asymmetry of ipsilateral motor cortex activation during unimanual motor tasks: further evidence for motor dominance. Clin Neurophysiol, 112 (2001), pp. 107-113

$38 \bullet \bullet$ S.A.L. Jayasinghe, F.R. Sarlegna, R.A. Scheidt, R.L. Sainburg. The neural foundations of handedness: insights from a rare case of deafferentation. J Neurophysiol, 124 (2020), pp. 259267. This study demonstrated the critical role of proprioception in combining motor control mechanisms specialized to each hemisphere. The authors examined reaching behavior in each hand of a deafferented individual as well as in a group of controls. Experimental data as well as computer simulations showed that in the absence of proprioception, each hand behaves like a controller in isolation, with the dominant right hand drifting away from the target and the nondominant left hand producing large directional errors and oscillations at the target. These results provide support for a model of handedness that is heavily dependent on proprioception.

39 V. Yadav, R.L. Sainburg. Handedness can be explained by a serial hybrid control scheme. Neuroscience, 278 (2014), pp. 385-396

40 D.J. Serrien, R.B. Ivry, S.P. Swinnen. Dynamics of hemispheric specialization and integration in the context of motor control. Nat Rev Neurosci, 7 (2006), pp. 160-166

41 V. Yadav, R.L. Sainburg. Limb dominance results from asymmetries in predictive and impedance control mechanisms. PLoS One, 9 (2014), Article e93892

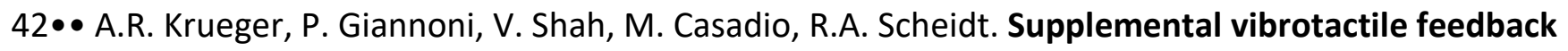
control of stabilization and reaching actions of the arm using limb state and position error encodings. J Neuroeng Rehabil, 14 (2017), p. 36. This study demonstrated the utility of artificial kinesthetic feedback to enhance stabilization and reaching tasks performed with the arm. The authors compared the objective and subjective utility of two forms of supplemental feedback of hand movement (limb state feedback and hand position error feedback) provided by a multi- 
channel vibrotactile feedback system. A series of human subjects experiments showed that both encoding schemes enhanced stabilization and reach performance in the absence of vision although error encoding yielded superior objective and subjective outcomes due to the additional task-relevant information it contains. The findings have implications for the development of sensory augmentation technologies for improving sensorimotor function after neuromotor injury in people who retain motor capacity but lack proprioceptive integrity in their more affected arm.

43 R.A. Andersen, T. Aflalo, S. Kellis. From thought to action: the brain-machine interface in posterior parietal cortex. Proc Natl Acad Sci U S A, 116 (2019), pp. 26274-26279

44 E. Okorokova, J.M. Goodman, N. Hatsopoulos, S.J. Bensmaia. Decoding hand kinematics from population responses in sensorimotor cortex during grasping. J Neural Eng, 17 (2020)

45 G. Valle, E. D’Anna, I. Strauss, F. Clemente, G. Granata, R. Di lorio, M. Controzzi, T. Stieglitz, P.M. Rossini, F.M. Petrini, et al. Hand control with invasive feedback is not impaired by increased cognitive load. Front Bioeng Biotechnol, 8 (2020)

46 G. Mochizuki, A. Centen, M. Resnick, C. Lowrey, S.P. Dukelow, S.H. Scott. Movement kinematics and proprioception in post-stroke spasticity: assessment using the Kinarm robotic exoskeleton. J Neuroeng Rehabil, 16 (2019), p. 146 